\title{
Níveis Bioquímicos após suplementação em Policias Militares durante treinamento físico
}

\author{
Biochemical levels in Military Police during Physical training after \\ supplementation
}

\author{
Celismar Lázaro da Silveiraํㅜ, Thiago Siqueira Paiva de Souza2*, Adenilson Targino de Araújo \\ Júnior ${ }^{3,4}$, Gilmário Ricarte Batista ${ }^{2}$, Monica da Silveira Torres ${ }^{4}$, Maria do Socorro Cirilo- \\ Sousa $^{2}$, Nuno Domingo Garrido ${ }^{1,5}$
}

ARTIGO ORIGINAL | ORIGINAL ARTICLE

\begin{abstract}
Este estudo analisou o efeito da suplementação com creatina ou glutamina sobre variáveis bioquímicas em policiais de militares. Neste propósito, desenvolveu-se um estudo experimental, duplo cego, com amostra de 32 homens distribuídos aleatoriamente, em três grupos: suplementados em creatina $(n=10)$, glutamina $(n=10)$ e grupo placebo $(n=12)$ e avaliados em três momentos distintos, num intervalo de três meses (T1, T2 e T3) durante o período do treinamento físico militar, com frequência de cinco sessões de 90 minutos, por semana. Por meio da análise sanguínea mensuraram-se: os níveis de creatinina, triglicerídeos, colesterol total, HDL, VLDL, LDL. Deste modo, foi observado que a suplementação não foi a principal responsável pelas modificações observadas nas medidas ao longo do estudo, já que apenas o efeito do tempo foi significativo em três medidas: CREA $\left(p=0.001, \eta_{\mathrm{p}}{ }^{2}=0.50\right)$, HDL $\left(p=0.001, \eta_{\mathrm{p}}{ }^{2}=0.30\right)$, LDL $(\mathrm{p}=0.009$, $\left.\eta_{\mathrm{p}}{ }^{2}=0.20\right)$. Ou seja, a suplementação com creatina ou glutamina em policiais militares submetidos ao treinamento físico militar não causou efeito sobre as medidas bioquímicas.

Palavras-chave: Suplementação alimentar, exercício, creatina, glutamina.
\end{abstract}

ABSTRACT

The objective of this study was to analyze the effect of the supplementation of creatine and glutamine on biochemical variables in military police officers. Therefore, an experimental double blind study was developed, with final sample composed by 32 men distributed randomly, in three groups: supplemented in creatine $(n=10)$, glutamine $(n=10)$ and placebo group $(n=12)$ and evaluated in three distinct moments, in an interval of three months (T1, T2 and T3). The physical training had weekly frequency of 05 sessions $\mathrm{x}$ 90 min of training. Through analysis of the blood is measured: the levels of creatinine, triglycerides, total cholesterol, HDL, VLDL, LDL. Thus, it was observed that supplementation was not primarily responsible for the changes observed in measures throughout the study, since only the time had a significant effect and in only three variables: CREA $\left(p=0.001, \eta_{p}{ }^{2}=0.50\right)$, HDL $\left(p=0.001, \eta_{p}{ }^{2}=0.30\right)$, LDL $\left(p=0.009, \eta_{p}^{2}=\right.$ 0.20 ). That is, supplementation with creatine or glutamine in the military police subjected to military physical training do not caused effect on biochemical measures.

Keywords: Supplementary feeding, Exercise, Creatine, Glutamine.

\footnotetext{
Artigo recebido a 04.06.2014; Aceite a 28.03.2016

${ }^{1}$ Universidade de Trás-os-Montes e Alto Douro, Vila Real, Portugal

${ }^{2}$ Laboratório de Cineantropometria e Desempenho Humano, LABOCINE/NPCMH/CCS/UFPB, Paraíba, Brasil

${ }^{3}$ Instituto Federal da Paraíba, Paraíba, Brasil

${ }^{4}$ Universidade Estadual do Rio de Janeiro, Rio de Janeiro, Brasil

${ }^{5}$ Centro de Investigação em Desporto, Saúde e Desenvolvimento Humano, CIDESD, Portugal

* Autor correspondente: Rua Manoel da Silva, 358, AP-302, Bairro Pedro Perazzo, CEP 58397-000, Areia, Paraíba, Brasil. Universidade Federal da Paraíba. E-mail: sps.thiago@gmail.com
} 


\section{INTRODUÇÃO}

Considerando os diferentes contextos sociais atuais, pode-se afirmar que a segurança pública se configura como um grave problema social, e que os profissionais que nela atuam, como os policiais militares, merecem atenção científica adequada (Santos Júnior, Dutra, \& Silva Filho, 2007). Dentre os fatores observados para melhoria da atuação profissional dos militares está o desempenho físico destes. Deste modo ressalta-se a importância de estudos que avaliem as mudanças que ocorrem nos militares em resposta ao Treinamento Físico Militar (TFM), que busca a melhoria da Aptidão Física, proporcionando um aumento significativo na prontidão desses profissionais para o combate.

Atualmente pode-se utilizar de meios, com os policiais militares, para minimizar o cansaço promovido pelas altas cargas de treinamento $\mathrm{e} /$ ou aperfeiçoar as suas capacidades físicas. Isto ocorre por intermédio dos produtos caracterizados como recursos ergogênicos que são alimentos ou componentes alimentares considerados capazes de aumentar a capacidade que um indivíduo tem de realizar um determinado exercício físico (Maughan \& Burke, 2004; Williams, 1983). Dentre a variedade de suplementos comercializados no mercado atual, pode-se mencionar a creatina (CR) e glutamina (GL), que tiveram sua ação ergogênica testada e comprovada no aumento dos níveis de força e potência muscular (Bemben \& Lamont, 2005; Gualano et al., 2008; Terjung et al., 2000; Wright, Grandjean, Pascoe, 2007) e defesa antioxidante (Kumar \& Annadan, 2007). Além disto, tem-se investigado a estabilização e melhorias em variáveis da bioquímica sanguínea (Sakkas et al., 2009; Volek et al., 1999; Volek et al., 2000).

Entretanto, as dosagens inadequadas podem não promover os efeitos esperados e ainda podem ser prejudicais a saúde (Hirschbruch \& Carvalho, 2002; Pedrosa, Qasen, Silva, \& Pinho, 2010). Nesse foco, pouco se sabe a respeito dos efeitos destes recursos suplementares sobre variáveis bioquímicas como os níveis de creatinina (CREA), triglicerídeos (TG), colesterol total (CT), lipoproteína da alta densidade (HDL), lipoproteína de muito baixa densidade (VLDL) e lipoproteína da baixa densidade (LDL), em uma amostra de policiais submetidos ao rigoroso treinamento físico militar.

Nesta perspetiva, este estudo analisou o desempenho de variáveis bioquímicas, obtidas pela análise de sangue, como os níveis de CREA, TG, CT, HDL, VLDL e LDL, em resposta à suplementação com creatina ou glutamina em militares. Apresentando a hipótese experimental de que a suplementação com esses aminoácidos promoveria alterações positivas nas medidas bioquímicas após três meses de treinamento físico.

\section{MÉTODO}

O estudo caracterizou-se como experimental, com processo amostral não probabilístico e abordagem longitudinal. Participaram do estudo policiais militares do estado de Tocantins-Brasil, integrantes do curso de formação de oficiais, fisicamente ativos, aparentemente saudáveis. Neste estudo foi utilizado o modelo duplo cego, onde os técnicos responsáveis não sabiam quais indivíduos faziam parte do Grupo Creatina, Grupo Glutamina ou Placebo, e a aleatoriedade, realizado por meio de sorteio, para a administração dos suplementos e formação dos grupos experimental e placebo.

\section{Participantes}

Para seleção dos participantes foi realizado cálculo amostral com base no erro de estimação $\alpha$ de $5 \%$, estimativa do tamanho do efeito de 0,5 , o poder $\beta$ de $80 \%$ e intervalo de confiança de $95 \%$ por meio do software estatístico Gpower, perfazendo um número de 36 sujeitos, sendo distribuídos aleatoriamente em três grupos: um que recebeu a suplementação de creatina (GCr, $\mathrm{n}=12$ ), outro com glutamina (GGL, $\mathrm{n}=12$ ) e o grupo placebo (GPL, $n=12$ ).

Todos os participantes assinaram ao Termo de Consentimento Livre e Esclarecido (TCLE), após serem informados sobre os procedimentos da pesquisa. O estudo recebeu a aprovação do Comitê de Ética em Pesquisa do Centro de Ciências da Saúde da Universidade Federal da Paraíba, protocolado sob $n^{\circ} 0312 / 2011$. Para inclusão no estudo foram estabelecidos os seguintes critérios: ter idade entre 18 e 30 anos, não fumar, não ingerir bebidas alcoólicas, não 
estar fazendo uso de esteroides anabolizantes, assim como de suplementos nutricionais de qualquer ordem, e responder negativamente a todos os itens do questionário Physical Activity Readiness Questionnaire (PAR-Q). Foram excluídos aqueles que ingeriram de forma indevida a suplementação proposta, realizassem sessões de treinamento físico nos dias que antecedessem as avaliações, não comparecessem nas datas marcadas para a realização das avaliações ou não cumprissem as atividades do Treinamento Físico Militar (TFM).

\section{Procedimentos}

As avaliações foram realizadas em uma sala de apoio e pista de campo na Academia de Policia Militar do Tocantins. Os grupos foram avaliados em três momentos de coletas distintos, em um período de três meses, a Avaliação prétreinamento (T1), após seis semanas de TFM (T2) e ao final do TFM (T3).

\section{Protocolo de treinamento}

O TFM foi realizado por 12 semanas, cinco vezes por semana, com duração de 90 minutos por sessão. Cada sessão foi constituída por quatro fases: alongamento, aquecimento, execução dos exercícios físicos e volta à calma. Os exercícios realizados nas sessões de treino eram compostos por exercícios aeróbios (corrida), calisténicos e atividades desportivas. O controlo das atividades foi realizado pelo próprio comando militar e supervisão do pesquisador, uma vez que neste ambiente o planejamento é cumprido em sua total exigência.

A suplementação foi utilizada sob a supervisão de um nutricionista. A creatina e a glutamina foram baseadas em protocolos já experimentados em estudos científicos (Cox, Mujika, Tumilty \& Burke, 2002; Deutekom, Beltman, Ruiter, Koning \& Haan, 2000; Edwards, Rhodes, Mackenzie \& Belcastro, 2000; Okudan \& Gokbel, 2005). Os suplementos foram administrados via oral dissolvidos em meio líquido adoçado (150 a 300ml), em dose de adaptação (primeira semana) de 0.3 g. $\mathrm{kg}^{-1}$ dividida em três doses diárias iguais e distribuídas ao longo do dia, e a dose de manutenção ( $\approx 12$ semanas) de $0.03 \mathrm{~g} \cdot \mathrm{kg}^{-1} \mathrm{em}$ dose única, 30min após o TFM. Todos os suplementos foram administrados em forma de sache. A ingestão foi realizada pela manhã no horário das 6h30min, antes de iniciar as atividades de TFM, sendo suplementados durante 93 dias sem interrupção.

O Placebo, constituído de amido de milho, também foi entregue na forma de sachês, acompanhando os mesmos procedimentos aplicados à suplementação de Creatina e Glutamina. É pertinente destacar que junto à suplementação foi adicionado um carboidrato de sabor artificial de fruta a mistura, para mascarar a presença dos suplementos nos sachês.

\section{Medidas}

A coleta do material para exames laboratoriais foi realizada por um profissional de enfermagem da Polícia Militar do Tocantins, Brasil. As coletas ocorreram sempre no turno da manhã, com aproximadamente 5 minutos de duração por indivíduo, feito a partir da coleta de $10 \mathrm{ml}$ de sangue retirados através de uma simples punção venosa periférica. $\mathrm{O}$ pesquisador acompanhou todo o processo, prezando pela segurança do material biológico obtido. Todos os procedimentos básicos pré-testes foram informados e respeitados por parte da amostra.

Após serem colhidas, as amostras foram analisadas em um laboratório adequado para realização das análises sanguíneas, sendo respeitadas nesse processo as normas técnicas e de vigilância sanitária. Determinou-se os níveis séricos ( $\mathrm{mg} / \mathrm{dL})$ de creatinina (CREA), triglicerídeos (TG), colesterol total (CT), HDL (Lipoproteína de alta densidade), VLDL (Lipoproteína de muito baixa densidade), LDL (Lipoproteína de baixa densidade).

Os hábitos alimentares da amostra foram avaliados a partir do recordatório habitual da dieta (Fisberg, Marchioni \& Colucci, 2009), o qual permite estimar os alimentos e as respetivas porções consumidas durante a pesquisa. Para o cálculo da estimativa de consumo energético diário os dados foram tratados de forma a serem corrigidos para a base/dia no programa VIRTUAL NUTRI (Philippi, Szarfarc \& Latterza, 1996) gerando valores do consumo energético 
total diário em quantidades consumidas para cada macronutriente (em gramas).

\section{Análise estatística}

Os dados foram analisados no SPSS para Windows versão 20.0. Os valores foram expressos em média e desvio padrão (DP). Antes de realizar a análise inferencial, foram testados os pressupostos de homogeneidade (Teste de Levene), para estatística univariada (Anova one way, post hoc Tukey), e esfericidade (Teste de Mauchly), para análise de medidas repetidas univariada (Anova repetead measure, post hoc Tukey), e no caso da ausência da esfericidade foi adotada a correção de Greenhouse-Geiser. A análise univariada de medidas repetidas $(3 \times 3)$ foi utilizada para averiguar o efeito do tempo (medidas: T1 $\times \mathrm{T} 2 \times \mathrm{T} 3$ ), do grupo (placebo $\times$ creatina $\times$ glutamina), e a interação (tempo $\times$ grupo), sendo utilizados os valores de tamanho de efeito do eta parcial quadrado $\left(\eta_{\mathrm{p}}^{2}\right)$, com valores entre $0.01,0.06$ e 0.14 representando pequeno, médio e alto efeito, respetivamente (Cohen, 1988). Em separado, o teste de anova univariada foi utilizada para verificar possíveis diferenças entre os grupos (GPL $\times \mathrm{GRr} \times \mathrm{GGL})$, para cada etapa de coleta (teste intergrupos), bem como, as possíveis alterações intragrupos entre as medidas $(\mathrm{T} 1 \times \mathrm{T} 2 \times \mathrm{T} 3)$. Em adição, foram apresentados os respectivos deltas de variação $(\% \Delta)$ entre a primeira e segunda $(\% \Delta 1)$, e entre a segunda e terceira $(\% \Delta 2)$ coletas. O nível de significância adotado foi de $5 \%$.

\section{RESULTADOS}

A análise de medidas repetidas (Tabela 1) para as medidas bioquímicas indicou haver um efeito significativo do tempo $(\mathrm{p}=0.001)$ sobre os valores de creatinina (CREA), com um alto tamanho do efeito. Ainda, para esta variável, as diferenças aparecem entre os testes realizados na primeira (T1) e segunda (T2) $(\mathrm{p}=0.001)$, e entre a primeira e terceira semana $(\mathrm{T} 3)(\mathrm{p}=0.001)$, existindo uma diminuição destas medidas ao final do estudo, principalmente entre $\mathrm{T} 1$ e $\mathrm{T} 2$ (Tabela 2). Continuando com a CREA foi observado na anova univariada que o grupo placebo (PL) $\left(\mathrm{F}_{(2,33)}=10.801, \mathrm{p}=0.001, \eta_{\mathrm{p}}{ }^{2}=\right.$ $0.40)$ apontou diferenças entre $\mathrm{T} 1$ vs $\mathrm{T} 2(\mathrm{p}=$ $0.001), \mathrm{T} 1_{\text {vs }} \mathrm{T} 3(\mathrm{p}=0.002)$ (Tabela 2$)$. O grupo suplementado com glutamina (GL) $\left(\mathrm{F}_{(2,27)}=\right.$ 4.615, $\left.\mathrm{p}=0.019, \eta_{\mathrm{p}}{ }^{2}=0.25\right)$ mostrou alterações significativas apenas entre o primeiro e último teste $(\mathrm{p}=0.025)$. Quando comparadas as medidas da CREA de cada etapa da coleta entre grupos foi verificada diferença significativa apenas para a terceira medida (T3) $\left(\mathrm{F}_{(2,29)}=\right.$ 3.646, $p=0.039, \eta_{p}^{2}=0.20$ ) entre os grupos GL e PL ( $\mathrm{p}=0.032)$, e como não houve diferença para a T1 afirma-se os grupos partiram da mesma condição. Voltando para análise de medidas repetidas, adiciona-se que $\mathrm{o}$ grupo não demonstrou ter influência sobre a CREA, visto que não houve efeito significativo, contudo, houve um efeito interativo do grupo com tempo $(\mathrm{p}=0.001)$.

Outra variável que também apresentou efeito do tempo (Tabela 1) foi a lipoproteína de alta densidade (HDL) $(p=0.001)$, com alto efeito, as disparidades apareceram entre todas as etapas, inicial (T1) e segunda (T2) $(\mathrm{p}=0.026), \mathrm{T} 1 \mathrm{e}$ T3 $(\mathrm{p}=0.001)$ e T2 e T3 $(\mathrm{p}=0.017)$. Houve uma diminuição nos níveis desta medida com o decorrer do estudo (Tabela 2). Porém não foram observadas alterações entre as medidas por cada grupo (teste intragrupo), bem como, entre os grupos (intergrupos). O efeito interativo e do grupo não foram significantes.

Para a medida bioquímica da concentração da lipoproteína de baixa densidade (LDL), foi verificado que o tempo $(p=0.009)$ exerceu alto efeito (Tabela 1). Houve um aumento das concentrações desta variável ao longo da pesquisa (Tabela 2), mais precisamente entre a medida inicial e as duas sequentes ( $\mathrm{T} 1$ vs $\mathrm{T} 2, \mathrm{p}=0.023$, $\left.\mathrm{T} 1_{\text {vs }} \mathrm{T} 3, \mathrm{p}=0.009\right)$. Entretanto, estas diferenças não aparecem para os testes intergrupos para cada fase pesquisa, e intragrupos, quando confrontadas as medidas entre si. Esta variável também não apresentou efeito do tipo de intervenção aplicada (efeito grupo), e não houve interação entre os grupos (PL, CR, GL) e as etapas (T1, T2 e T3). 
Tabela 1

Análise multivariada das variáveis bioquímicas ao longo dos três meses de estudo ( $n=32)$.

\begin{tabular}{|c|c|c|c|c|c|c|}
\hline \multicolumn{4}{|c|}{$\mathrm{CREA}_{(\mathrm{m} g / \mathrm{dL})}$} & \multicolumn{3}{|c|}{$\mathrm{HDL}(\mathrm{mg} / \mathrm{dL})$} \\
\hline Efeito estatístico & $\mathrm{F}$ & $\eta_{\mathrm{p}}^{2}$ & post hoc & $\mathrm{F}$ & $\eta_{\mathrm{p}}^{2}$ & post hoc \\
\hline Tempo (T) & $28.89 *{ }^{*}(2,58)$ & 0.50 & $\mathrm{~T} 1 \neq \mathrm{T} 2 \mathrm{~T} 1 \neq \mathrm{T} 3$ & $11.97^{*}(2,58)$ & 0.30 & $\mathrm{~T} 1 \neq \mathrm{T} 2 \mathrm{~T} 1 \neq \mathrm{T} 3 \mathrm{~T} 2 \neq \mathrm{T} 3$ \\
\hline Grupo (G) & $2.16_{(2,29)}$ & 0.10 & - & $0.58_{(2,29)}$ & 0.04 & - \\
\hline Interação T x G & $6.39 *(4,58)$ & 0.30 & - & $0.36_{(4,58)}$ & 0.02 & - \\
\hline \multicolumn{4}{|c|}{$\mathrm{LDL}_{(\mathrm{mg} / \mathrm{dL})}$} & \multicolumn{3}{|c|}{$\mathrm{VLDL}_{(\mathrm{mg} / \mathrm{dL})}$} \\
\hline Efeito estatístico & $F^{\varepsilon}$ & $\eta_{p}^{2}$ & post hoc & $\mathrm{F}$ & $\eta_{\mathrm{p}}^{2}$ & post hoc \\
\hline Tempo (T) & $5.72^{*}(1.61,46.81)$ & 0.20 & $\mathrm{~T} 1 \neq \mathrm{T} 2 \mathrm{~T} 1 \neq \mathrm{T} 3$ & $3.02(2,58)$ & 0.10 & - \\
\hline Grupo (G) & $0.81_{(2,29)}$ & 0.01 & - & $0.80_{(2,29)}$ & 0.05 & - \\
\hline Interação T x G & $0.09_{(3,22,46,81)}$ & 0.05 & - & $1.10_{(4,58)}$ & 0.05 & - \\
\hline & \multicolumn{3}{|c|}{ TG (mg/dL) } & \multicolumn{3}{|c|}{$\mathrm{CT}_{(\mathrm{m} / \mathrm{dL})}$} \\
\hline Efeito estatístico & $\mathrm{F}$ & $\eta_{\mathrm{p}}^{2}$ & post hoc & $F^{\varepsilon}$ & $\eta_{\mathrm{p}}^{2}$ & post hoc \\
\hline Tempo (T) & $1.425(2,58)$ & 0.05 & - & $0.73(1.61,46.81)$ & 0.02 & - \\
\hline Grupo (G) & $0.852_{(2,29)}$ & 0.08 & - & $2.35_{(2,29)}$ & 0.02 & - \\
\hline Interação T x G & $1.218_{(4,58)}$ & 0.05 & - & $0.29_{(3.22,46.81)}$ & 0.10 & - \\
\hline
\end{tabular}

Nota: CREA - creatinina; HDL - lipoproteína de alta densidade; LDL - lipoproteína de baixa densidade; VLDL - lipoproteína de muito baixa densidade; TG - triglicerídeos; CT - colesterol total; $\eta_{\mathrm{p}}{ }^{2}$ - Tamanho do efeito: 0.01, 0.06 e 0.14 representando pequeno, médio e alto efeito, respetivamente (Cohen, 1988). ${ }^{\varepsilon}$ Variável em que se adotou a correção de Greenhouse-Geisser; $\mathrm{p}<0.05$

Tabela 2

Variáveis bioquímicas nos grupos suplementados com creatina (CR, $n=10)$, glutamina $(G L, n=10)$ e placebo $(P L, n=12)$ antes, durante e após o período de intervenção.

\begin{tabular}{|c|c|c|c|c|c|}
\hline & $\begin{array}{c}\text { Início (T1) } \\
M \pm D P\end{array}$ & $\begin{array}{c}6 \text { semanas (T2) } \\
M \pm \mathrm{DP}\end{array}$ & $\begin{array}{c}12 \text { semanas (T3) } \\
\mathrm{M} \pm \mathrm{DP}\end{array}$ & $\begin{array}{c}\% \Delta 1 \\
(\mathrm{~T} 2-\mathrm{T} 1)\end{array}$ & $\begin{array}{c}\% \Delta 2 \\
(\mathrm{~T} 3-\mathrm{T} 2)\end{array}$ \\
\hline \multicolumn{6}{|l|}{ CREA (mg/dL) } \\
\hline Placebo & $1.19 \pm 0.12$ & $1.00 \pm 0.08$ & $1.03 \pm 0.09$ & $\downarrow 15.5$ & $\uparrow 2.7$ \\
\hline Creatina & $1.08 \pm 0.12$ & $1.05 \pm 0.11$ & $1.08 \pm 0.13$ & $\downarrow 2.9$ & $\uparrow 2.7$ \\
\hline Glutamina & $1.07 \pm 0.10$ & $0.97 \pm 0.08$ & $0.95 \pm 0.08$ & $\downarrow 9.4$ & $\downarrow 1.5$ \\
\hline \multicolumn{6}{|l|}{$\mathrm{HDL}_{(\mathrm{mg} / \mathrm{dL})}$} \\
\hline Placebo & $54.41 \pm 14.57$ & $49.92 \pm 9.55$ & $48.75 \pm 8.14$ & $\downarrow 8.3$ & $\downarrow 2.4$ \\
\hline Creatina & $51.24 \pm 13.21$ & $47.10 \pm 14.35$ & $42 \pm 10.95$ & $\downarrow 8.1$ & $\downarrow 10.8$ \\
\hline Glutamina & $54.37 \pm 9.38$ & $50.45 \pm 8.18$ & $46.1 \pm 6.2$ & $\downarrow 7.2$ & $\downarrow 8.6$ \\
\hline \multicolumn{6}{|l|}{$\mathrm{LDL}_{(\mathrm{mg} / \mathrm{dL})}$} \\
\hline Placebo & $81.48 \pm 25.45$ & $90.5 \pm 16.9$ & $92.61 \pm 22.51$ & $\uparrow 11.1$ & $\uparrow 2.3$ \\
\hline Creatina & $75.72 \pm 18.38$ & $83.28 \pm 16.74$ & $83.42 \pm 10.6$ & $\uparrow 10.0$ & $\uparrow 0.2$ \\
\hline Glutamina & $84.23 \pm 32.36$ & $93.81 \pm 22.73$ & $96.82 \pm 26.6$ & $\uparrow 11.4$ & $\uparrow 3.2$ \\
\hline \multicolumn{6}{|l|}{$\operatorname{VLDL}_{(\mathrm{mg} / \mathrm{dL})}$} \\
\hline Placebo & $15.6 \pm 5.03$ & $18.65 \pm 8.34$ & $19.05 \pm 10.16$ & $\uparrow 19.6$ & $\uparrow 2.1$ \\
\hline Creatina & $16.94 \pm 5.94$ & $20.96 \pm 8.13$ & $19.8 \pm 6.04$ & $\uparrow 23.7$ & $\downarrow 5.2$ \\
\hline Glutamina & $21.0 \pm 7.96$ & $19.54 \pm 7.2$ & $23.6 \pm 9.52$ & $\downarrow 7.0$ & $\uparrow 20.8$ \\
\hline \multicolumn{6}{|l|}{$\mathrm{TG}(\mathrm{mg} / \mathrm{dL})$} \\
\hline Placebo & $78 \pm 25.15$ & $101.58 \pm 53.63$ & $95.25 \pm 50.81$ & $\uparrow 30.2$ & $\downarrow 6.2$ \\
\hline Creatina & $94.7 \pm 49.45$ & $105.1 \pm 41.14$ & $99.4 \pm 30.24$ & $\uparrow 11.0$ & $\downarrow 5.4$ \\
\hline Glutamina & $111.2 \pm 47.4$ & $102.7 \pm 27.8$ & $123.4 \pm 54.8$ & $\downarrow 7.6$ & $\uparrow 20.2$ \\
\hline \multicolumn{6}{|l|}{$\mathrm{CT}_{(\mathrm{mg} / \mathrm{dL})}$} \\
\hline Placebo & $154.83 \pm 18.86$ & $160.75 \pm 17.59$ & $160.41 \pm 21.5$ & $\uparrow 3.8$ & $\downarrow 0.2$ \\
\hline Creatina & $145.9 \pm 23.25$ & $151.4 \pm 25.52$ & $145.3 \pm 15.93$ & $\uparrow 3.8$ & $\downarrow 4.0$ \\
\hline Glutamina & $163.3 \pm 27.3$ & $164.8 \pm 22.92$ & $167.6 \pm 23.76$ & $\uparrow 0.9$ & $\uparrow 1.7$ \\
\hline
\end{tabular}

Legenda: CREA - creatinina; HDL - lipoproteína de alta densidade; LDL - lipoproteína de baixa densidade; VLDL - lipoproteína de muito baixo densidade; TG - triglicerídeos; CT - colesterol total.

As demais variáveis bioquímicas, o VLDL, TG e CT não apresentaram efeito do tempo, do grupo e interação. Na medida da lipoproteína de muito baixa densidade (VLDL), houve variações entre as coletas (Tabela 2), no entanto, enquanto os grupos PL $(\% \Delta 1=20)$ e CR $(\% \Delta 1=24)$ aumentaram os valores da primeira para segunda etapa, o grupo CR (\% $\Delta 1=-7)$ diminuiu, ocorrendo o inverso de $\mathrm{T} 2$ para $\mathrm{T} 3$, tanto para o GL $(\% \Delta 1=21)$ quanto para o CR $(\% \Delta 1=-5,2)$.
$\mathrm{O}$ teste (anova univariada) não indicou diferenças entre os grupos para cada fase do estudo, o que foi afirmado pela ausência do efeito do grupo. As comparações entre as medidas para cada grupo em separado, da mesma forma, não apontaram diferenças significativas. Não houve efeito interativo para esta medida.

Os níveis de triglicerídeos (TG) não apresentaram nenhum tipo de efeito estatístico, já que o tempo, o grupo e a interação não 
resultaram em valores significativos. O mesmo ocorreu para a medida do colesterol total (CT), em que não foi identificado efeito do tempo, nem do tipo de intervenção suplementar, bem como, não houve efeito interativo dos grupos com as fases da pesquisa. Ademais, cita-se que para ambas as variáveis (CT e TG), não houve diferenças significativas para entre os grupos, para as medidas de primeira, segunda e terceira coletas, da mesma forma, que não existem alterações intragrupos quando confrontadas as medidas entre si.

A análise das variáveis mensuradas pelo Recordatório Habitual da Dieta, para a quantidade de calorias ingeridas (kcal) foi observado um alto efeito do tempo $\left(\mathrm{F}_{(1.61,46.71)}=\right.$ 10.060, $\left.\mathrm{p}=0.001, \eta_{\mathrm{p}}{ }^{2}=0.30\right)$, com as diferenças entre T1 e T2 ( $\mathrm{p}=0.002)$ e T1 e T3 $(\mathrm{p}=0.001)$. Houve uma queda da ingestão calórica com o decorrer da pesquisa. Ainda para esta variável, não foi observado o efeito do grupo, nem interação entre o grupo e o tempo. Os resultados não indicaram efeito do tempo, grupo ou interativo para quantidade (g) de proteína (PRT) ingerida. Para a quantidade (g) de carboidratos (CARB), o tempo causou um efeito significativo $\left(\mathrm{F}_{(1.294,37.535)}=11.000, \mathrm{p}=0.001, \eta_{\mathrm{p}}{ }^{2}=0.30\right)$ com as diferenças identificadas entre as medidas $\mathrm{T} 1_{\text {vs }} \mathrm{T} 2(\mathrm{p}=0.003)$ e $\mathrm{T} 1_{\text {vs }} \mathrm{T} 3(\mathrm{p}=0.001)$ havendo uma diminuição da ingestão deste nutriente entre a fase inicial e demais, para todos os grupos. O mesmo não ocorreu para o consumo de lipídios (LIP), em que não foi observado efeito do tempo, interativo ou do grupo.

\section{DISCUSSÃO}

A presente pesquisa teve como intuito analisar as alterações nas variáveis bioquímicas obtidas pela análise de sangue em resposta à suplementação com creatina ou glutamina em policiais de militares brasileiros. Mas, primeiramente apresentam-se alguns fatores que limitam as discussões dos resultados obtidos, como a falta de um controle alimentar rigoroso, dado que, as variáveis foram apenas mensuradas, não houve nenhum tipo de intervenção. Além disto, não foi mensurado os níveis de estresse da amostra, visto que, durante as coletas os mesmos estavam submetidos ao rigoroso treinamento físico militar.

Sendo assim, foi proposto como hipótese deste estudo que a suplementação de creatina e glutamina iria alterar os níveis bioquímicos de CREA, HDL, LDL, VLDL, TG e CT após três meses de treinamento físico. No entanto, a suplementação de creatina ou glutamina não foi a principal responsável pelas modificações observadas nestas variáveis, já que apenas o tempo apresentou efeito significativo, porém em apenas três medidas (CREA, HDL, LDL). Deste modo, rejeita-se a referida proposição.

Nesta investigação, e após uma análise inter e intragrupos, os níveis de creatinina no sangue diminuíram significativamente apenas para os grupos que foram suplementados com glutamina e placebo, enquanto os indivíduos que usaram a creatina (grupo CR) mantiveram as suas concentrações sem alterações durante todo estudo. Acredita-se que isto foi devido ao uma maior utilização dos estoques celulares de creatinafosfato $(\mathrm{CP})$ em resposta à carga de exercícios imposta, sem haver a devida reposição para os grupos (PL e GL) que não ingeriram a creatina exógena, sendo assim os militares que receberam a CR não apresentaram mudanças nas concentrações dos seus metabólitos. A degradação da fosfocreatina gera como produto final a creatinina (CREA) (Greenhaff, 2001).

Nesta linha de raciocínio, cita-se a pesquisa de Franco (2004), que verificou se protocolos de suplementação aguda ou crônica de creatina influenciariam a excreção de creatinina urinária dos animais (ratos) exercitados e sedentários. Conforme o autor a excreção de creatinina elevou-se após a ingestão aguda e crônica de creatina, independente do exercício. Em adição, salienta-se que nenhum indivíduo, independente do grupo, apresentou concentração de creatinina plasmática acima dos valores considerados normais, o que é um fator de risco para a doença renal crônica (Lessa, 2004; Rose \& Rennke, 1999). Em delineamento semelhante e com 18 futebolistas profissionais, Silva, Santhiago, Papoti e Gobatto (2006) mediu os avaliados no início ( $\mathrm{T} 1$, semana 0), meio (T2, semana 6) e fim (T3, semana 12) do treinamento. Os autores observaram um aumento dos níveis desta 
variável no sangue. Outros estudos não reportam alterações significativas nas concentrações séricas em resposta ao treinamento (Lehmann et al., 1991; Lehmann, Wiedland, \& Gastmann, 1997).

Um estudo, realizado por Prado et al. (2004), verificou o grau de correlação entre tais variáveis séricas em 12 cadetes da Academia Militar das Agulhas Negras (AMAN). De acordo com os resultados os autores observaram principalmente uma associação entre os níveis de colesterol total sanguíneo e o percentual calórico de lipídios total e lipídios saturados obtidos na dieta. Segundo o autor, para este tipo de amostra, um comportamento nutricional adequado é imprescindível, tanto para uma boa saúde, como para um bom desempenho físico (Prado et al., 2004). Fato que não ocorreu na presente pesquisa, já que a dieta foi apenas mensurada sem intervenção.

Nesta pesquisa, para as medidas bioquímicas que indicam as taxas lipídicas, a suplementação empregada nos militares brasileiros, não surtiu efeito. Cita-se a elevação ocorrida nas concentrações do LDL e redução do HDL que aconteceram com decorrer da pesquisa, e que foi similar em todos os grupos, desta forma, este efeito foi ocasionado apenas pela prática dos exercícios físicos obrigatórios durante o curso de formação de oficiais. Ademais, estas alterações nas concentrações de HDL e LDL não podem ser atribuídas à dieta dos militares, visto que, houve uma diminuição da ingestão calórica total (Kcal) para todos os grupos, sem diferença entre si. Conforme a literatura uma dieta rica em lipídios e colesterol representa um papel importante no desenvolvimento de doenças como dislipidemias e doença arterial coronariana (Caggiula \& Mustad, 1997; Nicolosi, Wilson, Lawton, \& Handelman, 2001; Schaefer, 2002), fato que não ocorreu neste estudo, dados os resultados mensurados pelo recordatório da dieta que não indicaram diferenças entre as fases de coleta para ingestão de lipídeos para os grupos suplementados (creatina ou glutamina) e placebo, sendo observada uma diminuição descritiva do consumo deste macronutriente em toda amostra.
Além disto, a quantidade de carboidrato (CARB) e lipídios (LIP) ingeridos foi menor ao final do estudo. Ou seja, as variáveis que poderiam influenciar o perfil lipídico da amostra, como por exemplo, com um aumento no consumo de gorduras e açúcar as taxas bioquímicas (LDL, VLDL e HDL) poderiam se alteradas, no entanto, não foi o que ocorreu, já que a ingestão alimentar diminui durante $\mathrm{o}$ experimento. Salienta-se que, apesar das alterações ocorridas nestas medidas, a amostra se encontra dentro dos padrões estabelecidos como normais pela literatura. Neste âmbito, destaca-se a importância de se enfatizar uma educação nutricional para promoção da saúde.

Embora o estudo, aqui realizado, tenha envolvido uma série de variáveis, ainda deve ser ampliado para a inserção de outras relacionadas à temática investigada. É pertinente que se incluam outros suplementos, um acompanhamento da rotina diária do militar e de alimentação, bem como o delineamento de fatores genéticos, no que se refere às variáveis bioquímicas. É possível que fatores psicológicos e emocionais interfiram e devam ser pesquisados, principalmente em relação às medidas qualitativas que permitem traçar o perfil do policial militar, no âmbito da sua atuação profissional. Também se faz necessário período maior de experimento, durante a preparação física, quantidade de medidas repetidas e padronização de atividades que permitam maior controle na intensidade.

\section{CONCLUSÕES}

O presente demonstrou que a suplementação com creatina ou glutamina em policiais militares submetidos ao treinamento físico militar não alterou as variáveis séricas: creatinina, lipoproteína de alta densidade (HDL), lipoproteína de baixa densidade (LDL), lipoproteína de muito baixa densidade (VLDL), triglicerídeos (TG) e colesterol total (CT). Isso implica em afirmar que o uso de Creatina e Glutamina, nas dosagens utilizadas nesse estudo, somado ao TFM é seguro ao usuário uma vez que promoveu a manutenção de variáveis da bioquímica sanguínea. 


\author{
Agradecimentos: \\ Academia da Polícia Militar do Estado de Tocantins
}

\section{Conflito de Interesses:}

Nada a declarar.

\section{Financiamento:}

Secretaria do Desenvolvimento Econômico, Ciência, Tecnologia e Inovação do Estado do Tocantins

\section{REFERÊNCIAS}

Bemben, M. G., \& Lamont, H. S. (2005). Creatine supplementation and exercise performance. Sports Medicine, 35(2), 107-125.

Caggiula, A. W., \& Mustad, V. A. (1997). Effects of dietary fat and fatty acids on coronary artery disease risk and total and lipoprotein cholesterol concentrations: epidemiologic studies. The American Journal of Clinical Nutrition, $65(5$ Suppl), 1597S-1610S.

Cohen, J. (1988). Statistical power analysis for the behavioral sciences. Hillsdale, New Jersey: Lawrence Erlbaum Associates.

Cox, G., Mujika, I., Tumilty, D., \& Burke, L. (2002). Acute creatine supplementation and performance during a field test simulating match play in elite female soccer players. International Journal of Sport Nutrition and Exercise Metabolism, 12(1), 33-46.

Deutekom, M., Beltman, J. G. M., Ruiter, C. J., Koning, J. J., \& Haan, A. (2000). No acute effects of shortterm creatine supplementation on muscle properties and sprint performance. European Journal of Applied Physiology, 82(3), 223-229.

Edwards, M. R; Rhodes, E. C., Mackenzie, D. C., \& Belcastro, A. N. (2000). The Effect of Creatine Supplementation on Anaerobic Performance in Moderately Active Men. Journal of Strength and Conditioning Research, 14(1), 75-79.

Fisberg, R. M., Marchioni, D. M. L., \& Colucci, A. C. A. (2009). Avaliação do consumo alimentar e da ingestão de nutrientes na prática clínica. Arquivos Brasileiros de Endocrinologia \& Metabolismo, 53(5): 617-624. doi: 10.1590/S0004-27302009000500014

Franco, F. S. C. (2004). Efeito da suplementação de creatina associada ou não á cafeína em ratos submetidos a exercício anaeróbico intermitente. Tese de Mestrado em Ciências da Nutrição. Universidade Federal de Viçosa, Viçosa, Brasil

Greenhaff, P. L. (2001). The creatine-phosphocreatine system: there's more than one song in its repertoire. Journal of Physiology, 537(3), 657.

Gualano, B., Benatti, F. B., Ferreira, J. C. B., Franchini, E., Brum, P. C., \& Lancha Júnior, A. H. (2008). Efeitos da suplementação de creatina no exercício intermitente de alta intensidade: divergências e recomendações metodológicas. Revista Brasileira de Cineantropometria e Desempenho Humano, 10(2), 189-196.

Hirschbruch, M. D., \& Carvalho, J. R. (2002). Nutrição Esportiva - uma visão prática. São Paulo: Manole.

Kumar H. S., \& Anandan R. (2007). Biochemical studies on the cardioprotective effect of glutamine on tissue antioxidant defense system in isoprenaline induced myocardial infarction in rats. Journal of Clinical Biochemestry and Nutrition, 4O(1), 49-55. doi: 10.3164/jcbn.40.49

Lehmann M., Dickhuth H. H., Gendrisch G., Lazar W., Thum M., \& Kaminsky R. (1991). Trainingovertraining: a prospective, experimental study with experienced middle and long distance runners. International Journal of Sports Medicine, 12(5), 444-452.

Lehmann M., Wiedland H., \& Gastmann U. (1997). Influence of an unaccustomed increase in training volume vs intensity on performance, hematological and blood-chemical parameters in distance runners. The Journal of Sports Medicine and Physical Fitness, 37(2), 110-116.

Lessa, I. (2004). Níveis séricos de creatinina: hipercreatininemia em segmento da população adulta de Salvador, Brasil. Revista Brasileira de Epidemiologia, $7(2), \quad 176-186 . \quad$ doi: 10.1590/S1415-790X2004000200007

Maughan, R. J., \& Burke, L. M. (2004). Nutrição esportiva: manual de ciência e medicina esportiva. Porto Alegre: Artmed.

Nicolosi, R. J., Wilson, T.A., Lawton, C., \& Handelman, G.J. (2001). Dietary effects on cardiovascular disease risk factors: beyond saturated fatty acids and cholesterol. The Journal of American College of Nutrition, 20 (5 Suppl), 421S-427S; discussion 440S-442S

Okudan, N., \& Gokbel, H. (2005). The effects of creatine supplementation on performance during the repeated bouts of supramaximal exercise. Journal of Sports Medicine and Physical and Fitness, 45(4), 507-511.

Pedrosa, O., Qasen, F., Silva, A., \& Pinho, S. (2011). Utilização de suplementos nutricionais por praticantes de musculação em academias da cidade de Porto Velho. Anais Da Semana Educa, 1(1). Recuperado Março 10, 2014, de http://www.periodicos.unir.br/index.php/sema naeduca/article/view/151/191

Philippi, S. T.; Szarfarc, S.; \& Latterza, A. (1996). Virtual nutri V 1.0 para Windows. São Paulo, [2 disquetes $3 \frac{1}{2}$ ]. Sistema de análise nutricional.

Prado, E. S., Peixoto, J. C., Silva, L. M. L., Pinheiro, J. C. S., Ferrão, M. L. D., Almeida, R. D., Melo, L. A., \& Dantas, E. H. M. (2004). Dieta lipídica e sua relação com os níveis séricos lipídicos/lipoprotéicos em cadetes da Academia Militar das Agulhas Negras (AMAN). Revista brasileira de Cineantropometria e Movimento Humano, 12(2), 57-62.

Rose B. D., \& Rennke H. G. (1999). Fisiopatologia Renal ( $1^{\mathrm{a}}$ ed.) Rio de Janeiro: Medsi. 
Sakkas G. K., Mulligan K., Dasilva M., Doyle J. W., Khatami H., Schleich T., Kent-Braun J. A., \& Schambelan M. (2009). Creatine fails to augment the benefits from resistance training in patients with HIV infection: a randomized, double-blind, placebo-controlled study. Plos One, 4(2):e4605. doi: 10.1371/journal.pone.0004605

Santos Júnior, A. A., Dutra L. H., \& Silva Filho D. B. (2007). Levantamento da percepção do medo e do crime em Santa Catarina. Revista Brasileira de Segurança Pública, 1(2), 94-119.

Schaefer, E. J. (2002). Lipoproteins, nutrition, and heart disease. The American Journal of Clinical Nutrition, 75(2), 191-212.

Silva, A. S. R., Santhiago, V., Papoti, M., \& Gobatto, C. A. (2006). Comportamento das concentrações séricas e urinárias de creatinina e uréia ao longo de uma periodização desenvolvida em futebolistas profissionais: relações com a taxa de filtração glomerular. Revista Brasileira de Medicina do Esporte, 12(6), 327-332.

Terjung, R. L., Clarkson, P., Eichner, E. R., Greenhaff, P. L., Hespel, P. J., Israel, R. G., Kraemer, W. J., Meyer, R. A; Spriet, L., Tarnopolsky, M. D., Wagenmakers, A. J. M., \& Williams, M .H. (2000). American College of Sports Medicine roundtable. The physiological and health effects of oral creatine supplementation. Medicine and Science in Sports and Exercise, 32(3), 706-717.

Volek J. S., Duncan N. D., Mazzetti S. A., Staron R. S., Putukian M., \& Gomez A. L. (1999). Performance and muscle fiber adaptations to creatine supplementation and heavy resistance training. Medicine \& Science in Sports \& Exercise, 31(8), 1147-1156.

Volek J. S., Duncan N. D., Mazzetti S. A., Putukian M., Gomez A. L., \& Kraemer W. J. (2000). No effect of heavy resistance training and creatine supplementation on blood lipids. International Journal of sport nutrition and exercise metabolism, 10(2), 144-156.

Williams, M. H. (1983). Ergogenic aids in sport. Champaign, Ill: Human Kinetics Publishers.

Wright, G. A., Grandjean, P. W., \& Pascoe, D. D. (2007). The effects of creatine loading on thermoregulation and intermittent sprint exercise performance in a hot humid environment. Journal of Strength and Conditioning Research, 21(3), 655-660.

Ziegler T. R., Benfell K., Smith R. J., Young L. S., Brown E., \& Ferrari-Baliviera E.(1990). Safety and metabolic effects of L-glutamine administration in humans. Journal of Parenteral and Enteral Nutrition, 14(4 Suppl), 137S-146S.

Todo o conteúdo da revista Motricidade está licenciado sob a Creative Commons, exceto quando especificado em contrário e nos conteúdos retirados de outras fontes bibliográficas. 